

\title{
Surface plasmon polaritons in sub-wavelength metallic gratings studied by higher-order angle-resolved spectroscopy
}

\author{
Camille Maxime, Jochen Fick
}

\section{- To cite this version:}

Camille Maxime, Jochen Fick. Surface plasmon polaritons in sub-wavelength metallic gratings studied by higher-order angle-resolved spectroscopy. European Physical Journal: Applied Physics, 2009, 48 (1), pp.10901. 10.1051/epjap/2009123 . hal-00504648

\section{HAL Id: hal-00504648 \\ https://hal.science/hal-00504648}

Submitted on 21 Jul 2010

HAL is a multi-disciplinary open access archive for the deposit and dissemination of scientific research documents, whether they are published or not. The documents may come from teaching and research institutions in France or abroad, or from public or private research centers.
L'archive ouverte pluridisciplinaire HAL, est destinée au dépôt et à la diffusion de documents scientifiques de niveau recherche, publiés ou non, émanant des établissements d'enseignement et de recherche français ou étrangers, des laboratoires publics ou privés. 


\title{
Surface plasmon polaritons in sub-wavelength metallic gratings
}

\section{studied by higher-order angle-resolved spectroscopy}

Camille Maxime and Jochen Fick

Institut Néel, CNRS and Université Joseph Fourier, BP 166, 38042 Grenoble, France

Received: date / Revised version: date

\begin{abstract}
Angle-resolved reflection spectroscopy is used to study surface plasmon polariton modes of metallic gratings consisting of sub-wavelength grooves. The used optical set-up allows recording of zero and $m=-1$ order reflection spectra in the visible and near infra-red spectral region. The different modes and their mutual coupling was identified in the dispersion diagram by means of modal theory. Surface plasmon modes at the grating surface and cavity modes in the sub-wavelength grooves were evidenced.
\end{abstract}

PACS. 42.25.Fx Diffraction and scattering - 78.67.-n Optical properties of low-dimensional, mesoscopic, and nanoscale materials and structures - 42.79.Dj Gratings

\section{Introduction}

Over the last years, surface plasmon-based devices are of growing interest for their potential applications in high sensitivity sensing [1,2], improved light sources [3] or in integrated optics devices[4]. Amongst different plasmonic systems such as metallic nano-particles or aperture arrays, metallic gratings are very efficient for coupling radiative light to surface plasmon polaritons (SSP) $[2,5-7]$. In the case of sub-wavelength grooves gratings, the coupling into cavity modes inside the grooves allows to realCorrespondence to: jochen.fick@grenoble.cnrs.fr ize very strong electromagnetic fields enhancement [8-12]. This effect is reinforced by the absence of the low energy cut-off for fundamental plasmon mode propagation in the one dimensional structure [13]. The grating parameters allow to adjust the wavelength and width of the plasmon resonances to the aimed application, such as coupling to luminescent materials.

The plasmon resonances can be experimentally studied by optical far field methods such as angle resolved reflectivity measurements $[14-16]$. This technique allows straightforward determination of the the cavity modes and 




Fig. 1. Schema of the angle-resolved reflection measurement set-up.

their interaction with the SSP. We have developed an experimental set-up for angle-resolved reflection measurements over a wide spectral and angular range. The possibility of recording higher order diffraction orders improves the interpretation of the experimental results.

Sub-wavelength metallic reflection and transmission gratings were theoretically studied using different approaches such as modal approaches with surface impedance boundary conditions [17-20] or exact solutions [21-24], transfer matrix approaches $[22,25]$, finite-difference time domain methods [26] or exact electromagnetic theory [27]. In this paper a straightforward modal model will be used for analysing our experimental data. It will be shown that even if some of its approximations become critical, it is sufficient for data exploitation.

\section{Experimental}

\subsection{Optical set-up}

The experimental set-up used for angle-resolved reflectivity measurements is shown on Fig. 1 . It is based on a $\theta / 2 \theta$ goniometer and a double beam, double monochromator spectrophotometer (Perkin Elmer Lambda 900). The two modules are linked by two multimode optical fibers. The output of the first fiber is imaged onto the sample by an optical system consisting of a two lens telescope, an iris, and a Glan-Taylor polarization prism. All measurements were made in TM (or p) polarisation. In the present case, the size $\sigma$ of the Gaussian beam and the aperture angle $\alpha$ were chosen to $\sigma=400 \mu m$ and $\alpha=4^{\circ}$. For small gratings the beam size can be reduced to $\sigma=100 \mu \mathrm{m}$. The sample is mounted in the centre of the home made $\theta / 2 \theta$ goniometer built by two concentric rotary stages with angular resolution of $0.01^{\circ}$. The reflected beam is coupled into the second fiber by a two lens telescope mounted on the goniometer branch.

The entire set-up is computer-controlled. Measurements of the zero order or specular reflection spectra $R^{(0)}$ and of higher order reflection spectra $\mathrm{R}^{(m)}$ are possible. In the case of $\mathrm{R}^{(0)}$ the incident angle range is limited to $5-85^{\circ}$. For higher order modes the angular range depends on the order. In general it consist of two lobes measured for forward and backward scattering geometry, respectively. All zero order spectra are normalized by the planar metallic surface reflection, measured beside the grating.

The dispersion diagrams are calculated from reflection spectra series by constructing a colour plot representing the reflected intensity as a function of the normalized in plane wave vector $k_{\|}=k_{0} d / \pi \sin \theta$ and the wave number $w n=1 / \lambda$. 


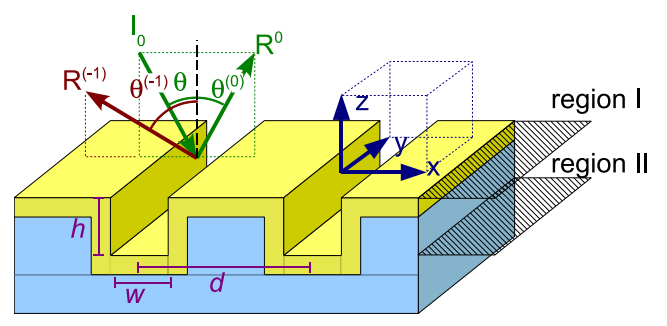

Fig. 2. Schema of investigated grating geometry.

\subsection{Sample fabrication}

Metal gratings with period $d$, groove height $h$ and width $w$ (Fig. 2) were fabricated by standard electron beam lithography [19]. A $\mathrm{SiO}_{2}$ layer was first thermally grown on a silicon wafer. After deposition of an electronegative resist, the gratings were directly written by e-beam. The irradiated resist and the $\mathrm{SiO}_{2}$ beneath were etched away. The resulting structured surface was metallised by thermal evaporation of a 60 - $100 \mathrm{~nm}$ thick gold or aluminium layer.

In this paper we present representative results from two gratings with period $d=600 \mathrm{~nm}$ and groove width $w=150 \mathrm{~nm}$ : a gold grating with $h=300 \mathrm{~nm}$ and an aluminium grating with $h=400 \mathrm{~nm}$.

\section{Theory}

Throughout this paper we will distinguish direct grating scattering modes $\mathrm{GM}^{(m)}$, surface modes $\mathrm{SM}^{(m)}$, and cavity modes $\mathrm{CM}^{(m)} \cdot \mathrm{GM}^{(m)}$ represents the $\mathrm{m}^{\text {th }}$-order grating scattering mode. Its scattering angle $\theta^{(m)}$ is defined by

$$
\sin \theta^{(m)}=\sin \theta+m \frac{\lambda}{d}
$$

$\theta$ and d being the incident angle and the grating period, respectively. The corresponding diffracted energy is labeled $\mathrm{R}^{(m)}$ (Fig. 2).

$\mathrm{SM}^{(m)}$ is the $\mathrm{m}^{\text {th }}$ surface plasmon polariton at the grating surface. Its dispersion can be calculated by

$$
k_{\|}^{S M}=k_{s p}\left(\sin \theta^{(m)}+m \frac{\lambda}{d}\right)
$$

with $k_{\|}^{S M}$ the in-plane component of the $\mathrm{SM}^{(m)}$ wave vector, $k_{s p}=k_{0} \sqrt{\epsilon_{m} /\left(1+\epsilon_{m}\right)}$ the surface plasmon wave vector at a plane metal surface, and $k_{0}=2 \pi / \lambda$ the vacuum wave vector. For good metals the dielectric constant $\left|\epsilon_{m}\right| \gg 1$. Thus $k_{s p}$ is only slightly greater than $k_{0}$ and $\mathrm{GM}^{(m)}$ and $\mathrm{SM}^{(m)}$ modes are very close. The coupling of the resonant SM contribution with the non-resonant contribution of direct scatterings explains the presence of Fano-type resonances with its asymmetric reflectivity profiles [28].

Finally, $\mathrm{CM}^{(m)}$ describes the $\mathrm{m}^{\text {th }}$ order cavity mode inside the grating grooves. These cavity modes are FabryPerot type modes built of vertically guided surface plasmons modes inside the grooves. Their resonance wavelengths are independent form the incident angle and can be approximated by [19]

$$
\lambda^{(m)}=\frac{4 h}{2 m-1},
$$

$\mathrm{h}$ being the grooves depth.

Pure SM and CM modes exist only under special conditions. In most cases the modes are of hybrid nature with contributions of at least two modes. Often one contribution is, however, dominant. For the sake of clarity the 
dominant mode will be used to label the actually hybrid mode.

The mode coupling and the dispersion of the resulting hybrid modes cannot be describe by Eq. (1)-(3). For this reason, we apply a straightforward modal model for the interpretation of the experimental results [19]. In this approach the electromagnetic field above the grooves is describe by a Rayleigh expansion (Fig. 2: region I). Inside the grating grooves (region II) the field is developed into a set of guided or evanescent eigenmodes. The air/ metal interfaces at the grating surface and at the bottom of the groves are described in the surface impedance model, whereas the groove walls are supposed to be of perfectly conducting metal.

This model allows to calculate the reflection spectra, the dispersion diagrams and the field and energy flow maps in the vicinity of the grating. The permittivity of gold and aluminium was taken as polynomial fit to av-

\section{1 zero-order reflection $\mathbf{R}^{(0)}$} eraged literature data [29]. It was shown that the model works very well in the infra-red spectral region [19]. In the visible, the perfect metal approximation becomes, however, critical. The penetration of the electromagnetic fields into the metal is not anymore negligible. The associated increase of the wave vector of the guided modes inside the grooves can be considered by an artificial overestimation of the groove depth. The cautious application of this correction allows to use the model for the interpretation of the experimental data.
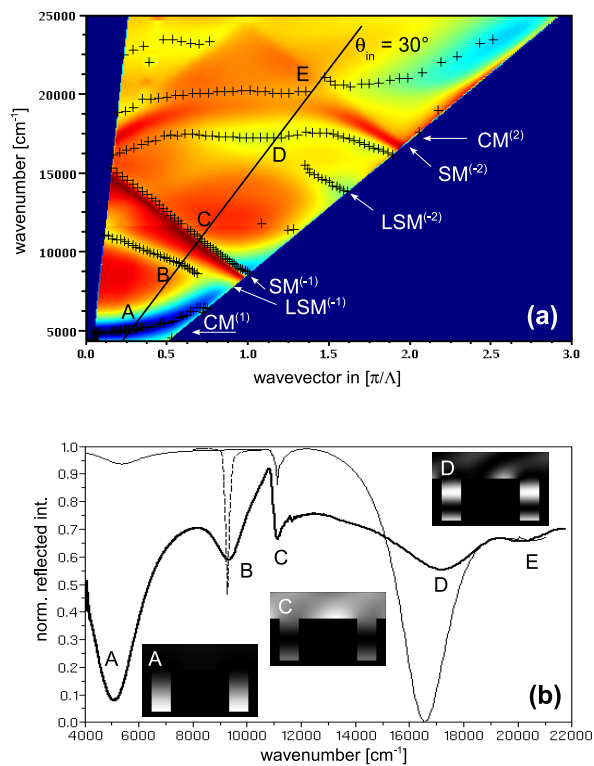

Fig. 3. (a) Gold grating experimental zero-order dispersion diagram (+: local minima). (b) Experimental (bold line) and theoretical reflection spectra for $\theta=30^{\circ}$ (thin line $\epsilon_{d}=\epsilon$ (air), dashed line $\left.\epsilon_{d}=\epsilon\left(\mathrm{SiO}_{2}\right)\right)$. The insets show calculated magnetic field intensity maps.

\section{Results and discussion}

The zero-order dispersion diagram of the gold and aluminium gratings are shown on Fig. 3.a and 4.a. The surface (SM) and cavity modes (CM) can be clearly distinguished by their dispersion. The SMs are represented by a network of inclined straight lines (Eq. (2)) whereas the horizontal lines correspond to CMs (Eq. (3)). These observable lines correspond to local minima of the zero order reflection due to intrinsic plasmon mode losses at the metal surface. 


\subsubsection{Gold grating}

In the case of the gold grating (Fig. 3), only the first order cavity mode $\mathrm{CM}^{(1)}$ is clearly visible as a broad horizontal line near $5000 \mathrm{~cm}^{-1}$. Due to coupling to the $\mathrm{SM}^{(0)}$ mode its dispersion curve upturns for high incident angles to approach asymptotically the light line $(k=\omega / c)$. Likewise, the $\mathrm{SM}^{(-1)}$ mode is the only $\mathrm{SM}$ mode which is mostly uncoupled and characterised by a straight dispersion line.

For a more detailed interpretation, the measured and calculated reflection spectra at an incident angle of $\theta=$ $30^{\circ}$ are presented in Fig. 3.b. Five minima, labeled A to E, can be distinguished. Due to their shape and their dispersion they can be attributed to coupling to first and second order plasmonic cavity modes $(A, D)$ and surface plasmon polaritons at the grating surface (C). The minimum E corresponds to the gold optical absorption edge near $500 \mathrm{~nm}$.

The main features of the experimental results are reproduced by the calculated spectrum. To match the positions A and D, a groove depth of $h=400 \mathrm{~nm}$ has, however, to be used instead of the nominal value of $h=300 \mathrm{~nm}$. This adjustment agrees with the fact that our model underestimates the cavity mode wavelengths. The difference in contrast can be explained by additional losses due to imperfect metal films. The theoretical magnetic field intensity maps for minimums A, C and D are shown as insets in the Fig 3.b.

The intensity map confirms the nearly pure first order cavity mode $\mathrm{CM}^{(1)}$ at $\mathrm{A}$. Modes $\mathrm{C}$ and $\mathrm{D}$ correspond to coupled modes with contributions of the second order cavity mode $\mathrm{CM}^{(2)}$ and the $\mathrm{SM}^{(-1)}$ and $\mathrm{SM}^{(-2)}$ surface modes, respectively. The SM (CM) mode contribution is majoritary in case of mode $\mathrm{C}(\mathrm{D})$. Mode $\mathrm{D}$ evolutes with increasing incident angle from an $\mathrm{SM}^{(1)} / \mathrm{CM}^{(2)}$ coupled mode to an $\mathrm{SM}^{(-2)} / \mathrm{CM}^{(2)}$ mode. In this case the $\mathrm{SM}$ contribution is majoritary for small and high incident angels, whereas the CM contribution is dominant for medial angles.

\subsubsection{Lower surface modes}

Minimum B is linked to the excitation of an SPP at the lower interface of the gold film. The excitation of this lower surface mode (LSM) is plausible as the metal penetration depth of a SPP on a gold/air interface is about $\delta_{m}=24 \mathrm{~nm}$ at $\lambda=1 \mu \mathrm{m}$. Moreover, coupling of the SPP modes on both sides of a $100 \mathrm{~nm}$ thick metal film was experimentally demonstrated [30]. The LSM modes cannot be described in the framework of our model. The position of the $\operatorname{LSM}^{(-1)}$ can, however, be confirmed by calculating the reflection of a silica covered gold grating (dashed lin in Fig 3.b).

The shape of minima B and C is different. The $\mathrm{SM}^{(-1)}$ line $\mathrm{C}$ shows the steep low energy slope characteristic for Fano type resonances due to coupling with the direct scattering mode $\mathrm{GM}^{(-1)}$. This coupling results also in the pronounced local reflection maximum on the low energy side of C. The LSM mode is more distant to GM modes. Its line shape is symmetric without any adjacent local maximum. The different line shapes of the SM and LSM modes are perceptible on the dispersion diagrams. 
In order to confirm experimentally the presence of SM and LSM modes, we measured the plasmon dispersion diagram of the gold grating immersed into water $(\mathrm{n} \approx 1.3)$ or an commercial index liquid $(\mathrm{n} \approx 1.5)$. In both cases, only the LSM dispersion lines remains unchanged. The other resonances (SM and $\mathrm{CM})$, related to plasmon modes at the metal top surface, shifted to lower wave numbers, according to the calculations.

\subsubsection{Aluminium grating}

No fundamental differences between the $\mathrm{Au}$ and $\mathrm{Al}$ grating dispersion can be observed. Due to the deeper grooves of the Al-grating, its $\mathrm{CM}^{(1)}$ mode appears just perceptible below $4000 \mathrm{~cm}^{-1}$, but cannot be revealed. Further on, gold has an absorption edge near $20000 \mathrm{~cm}^{-1}$. Consequently the gold plasmon resonances becomes indistinct above this energy. In the case of the aluminium grating the plasmon resonance lines stays distinct even for high wave numbers. Finally, two lower surface modes $\left(\operatorname{LSM}^{(-1)}\right.$ and $\left.\operatorname{LSM}^{(-2)}\right)$ are clearly visible.

\section{$4.2 m=-1$ order reflection}

The zero order reflection diagram allows to reveal the main features related to surface plasmon resonances in sub-wavelength metallic gratings. Higher order diagrams can, however, be useful to improve the insight into the coupling mechanisms of different resonances. The Al-grating was chosen for recording the $m=-1$ order reflection diagram, as aluminium is the better metal in the accessible
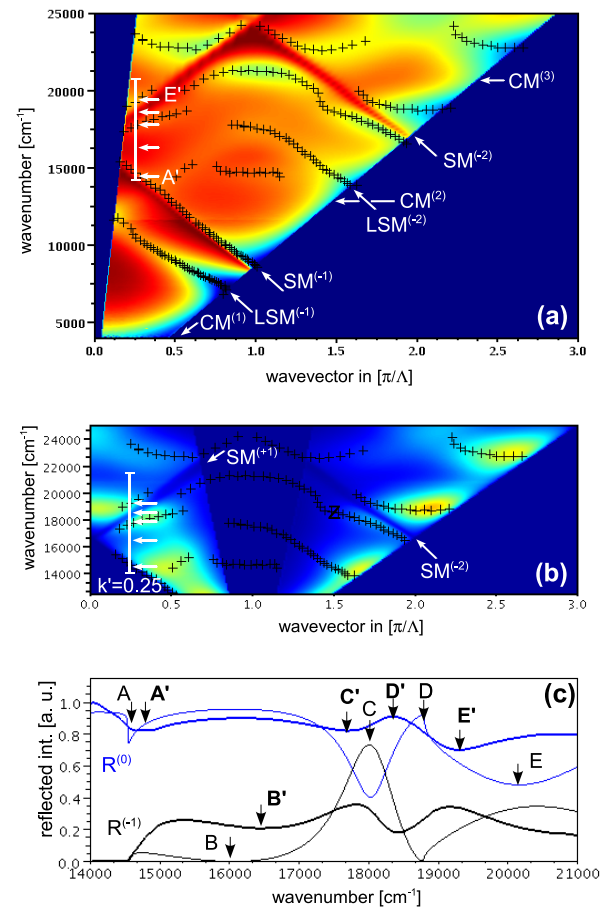

Fig. 4. Aluminium grating experimental zero-order (a) and $m=-1$ (b) order dispersion diagrams. The minima in $\mathrm{R}^{(0)}$ (crosses) were also reported on (b). (c) Experimental (bold line) and theoretical $\mathrm{R}^{(0)}$ and $\mathrm{R}^{(-1)}$ reflection spectra for $k^{\prime}=$ 0.25 as indicated in (a) and (b).

wavelength region of $\lambda=400-700 \mathrm{~nm}$. Its $\mathrm{R}^{(-1)}$ diagram is shown on Fig. 4.b. Because of the set-up geometry, only two lobes are accessible above the light line and the $\mathrm{GM}^{(-1)}$ limit. For comparison, the $\mathrm{R}^{(0)}$ minima positions are indicated by crosses. In general, they coincide with the maxima in $\mathrm{R}^{(-1)}$. Two straight dark lines are observed at the positions of the $\mathrm{SM}^{(+1)}$ and $\mathrm{SM}^{(-2)}$ modes.

Recording of the $m=-1$ reflection diagram requires that the $m=-1$ scattering mode is radiative, i.e. the right hand side of eq. 1 has to be $<1$. This condition limits the accessible wave number/ wave vector region to points above the $\mathrm{GM}^{(-1)}$ line, which virtually coincides 


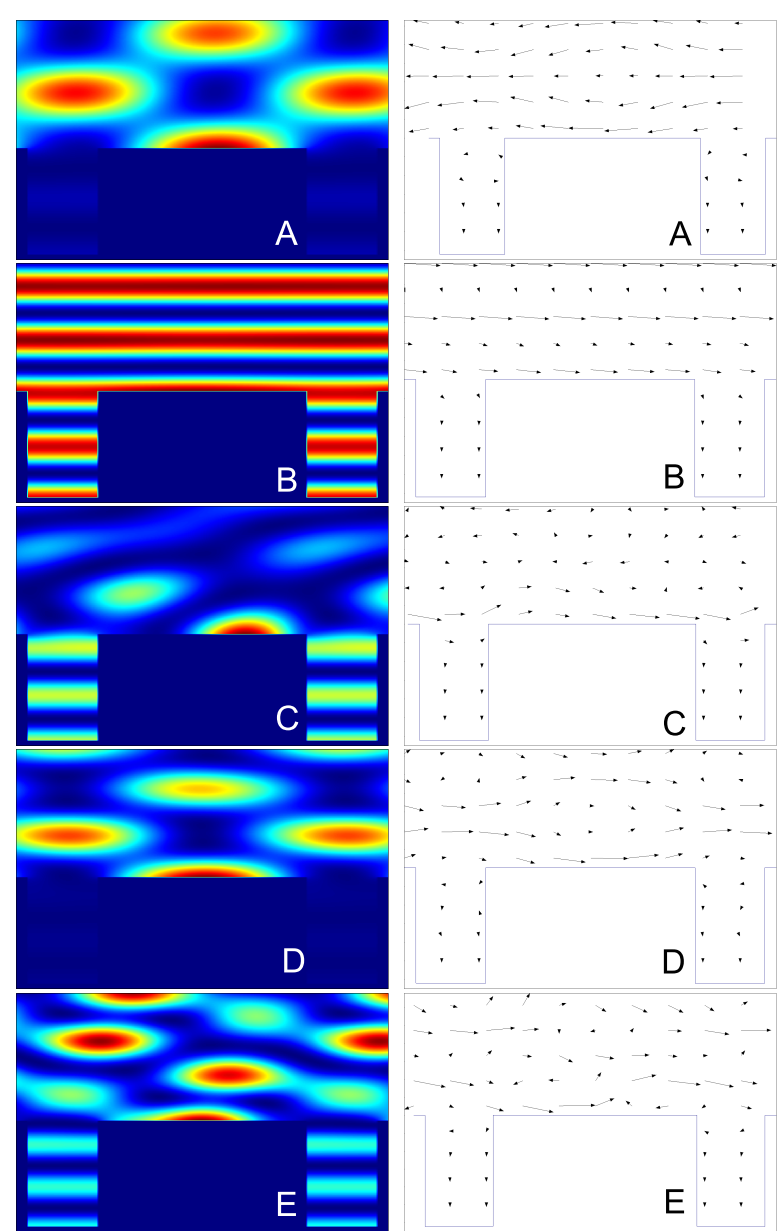

Fig. 5. Calculated magnetic field intensity (left) and energy flow maps (right) for points A-F as indicated in Fig. 4.

with the $\mathrm{SM}^{(-1)}$ line indicated in Fig. 4.a. For the region below this line, the $m=-1$ scattering mode is evanescent in the $z$-direction. Thus it cannot be detected with far field techniques, but can eventually couple to the SPP near-field modes.

For further interpretation the measured zero and $m=$ -1 order reflection spectra (bold lines) at constant normalized in-plane wave vector $k^{\prime}=0.25$ are shown and compared to calculated spectra (light lines) in Fig. 4.c. In this region the influence of lower surface modes (LSMs) is negligible. Five main features labeled A-E for the theoretical curves and A'-E' for the experimental ones are distinguishable on the figure. Similar to the case of the gold grating, the grooves depth used for the calculation was overestimated $(h=620 \mathrm{~nm})$ in order to match the experimental curves. Even if the agreement of the resonance positions could be improved, the general shape of the curves agrees very well. The interpretation of the experimental results is thus viable.

In general $\mathrm{R}^{(0)}$ and $\mathrm{R}^{(-1)}$ shows opposite behaviour, i.e. minima in $\mathrm{R}^{(0)}$ correspond to maxima in $\mathrm{R}^{(-1)}$ and vice versa. At A the grating scattering mode $\mathrm{GM}^{(-1)}$ opens, i.e. changes from near to far field. This effect is overlapped by coupling into the $\mathrm{SM}^{(-1)}$ plasmon mode. The coupling of the $\mathrm{GM}^{(-1)}$ and $\mathrm{SM}^{(-1)}$ modes results in a Fano type resonance, visible by the sequence of maxima and asymmetric minima in $\mathrm{R}^{(0)}$. The calculated magnetic field intensity map (Fig 5) indicates a first order mode with one lobe in-between two grating grooves. The negative order is revealed by energy flow into the $-x$ direction at the top of the grating.

The $\mathrm{R}^{(-1)}$ spectra shows a minima at $\mathrm{B}$, hardly to distinguish as maximum in $\mathrm{R}^{(0)}$. At this point the wavelength is equal to the groove depth. In the theoretical model the wave vector of the first order guided mode is equal to the free space wave vector $k_{0}$. Thus the light reflected at the bottom of the grooves has the same phase as the light reflected on top of the grating. Consequently, reflection of the incoming light is concentrated into the $\mathrm{R}^{(0)}$ order such as in the case of a plane metal surface (Fig 5.b). Even if this feature is closely related to the perfectly conducting 
groove walls approximation, it is distinguishable in the experimental curve.

Features $\mathrm{C}$ and $\mathrm{E}$ correspond to two hybrid modes, resulting from coupling of the $\mathrm{CM}^{(3)}$ cavity mode with the $\mathrm{SM}^{(+1)}$ surface mode. The intensity and energy flow maps correspond to hybrid modes of a $m=+1$ surface mode and a third order cavity mode. The minimum/ maximum at $\mathrm{D}$ could be explained as the interstice in between the resonances $\mathrm{C}$ and $\mathrm{E}$. Its intensity map shows, however, that the pure $\mathrm{SM}^{(+1)}$ mode exist at this position. This mode is orthogonal to the $\mathrm{SM}^{(-1)}$ mode, explaining the minimum in $\mathrm{R}^{(-1)}$. The absence of this diffraction order and the lower intrinsic losses of the pure surface mode compared to hybrid modes, result in the local peak of $\mathrm{R}^{(0)}$ with a steep high energy slope due to the scattering onset of $\mathrm{GM}^{(+1)}$.

$\mathrm{R}^{(-1)}$ dispersion diagrams were recorded for TE polarisation (not shown). They only show the slow angular evolution of the grating scattering efficiency. The fine structure, such as the $\mathrm{SM}^{(+1)}$ and $\mathrm{SM}^{(-2)}$ lines in TM case were absent, indicating that the observed features are due to the excitation of surface plasmon modes.

\section{Conclusions}

Plasmon resonances of sub-wavelength metallic gratings were studied by angle resolved reflection spectroscopy. Surface plasmon modes at the grating surface and cavity modes in the sub-wavelength grooves were evidenced in zero order spectroscopy dispersion diagrams. Lower surface plasmon modes at the metal-silica interface below the metal film were observed. The coupling of surface and cavity modes was investigated by zero and $m=-1$ order reflection diagrams. It was shown that the latter is useful to affirm and complete and the information of the zero order ones.

\section{References}

1. J. Anker, W. Hall, O. Lyandres, N. Shah, J. Zhao, R. Van Duyne, nature mater 7, 442 (2008)

2. X. Hoa, M. Martin, A. Jimenez, J. Beauvais, P. Charette, A. Kirk, M. Tabrizian, Biosens. Bioelectron. 24, 976 (2008)

3. T. Ozel, I. Soganci, S. Nizamoglu, I. Huyal, E. Mutugun, S. Sapra, N. Gaponik, A. Eychmueller, H. Demir, New J. Phys. 10, 083035 (2008)

4. E. Ozbay, Science 311, 189 (2006)

5. C. Symonds, C. Bonnand, J. Plenet, A. Bréhier, R. Parashkov, J. Lauret, E. Delporte, J. Bellessa, New. J. Phys. 10, 065017 (2008)

6. J. Gomez Rivas, G. Vecchi, V. Giannini, N. J. Phys. 10, 105007 (2008)

7. P. Andrew, W. Barnes, Phys. Rev. B 64, 125405 (2001)

8. G. Winter, W. Murray, S. Wedge, W. Barnes, J. Phys.: Condens. Matter 20, 304218 (2008)

9. Z. Ruan, M. Qiu, Appl. Phys. Lett. 90, 201906 (2007)

10. A. Barbara, J. Le Perchec, P. Quémerais, T. Lopez-Rios, N. Rochat, J. Appl. Phys. 98, 033705 (2005)

11. P. Quémerais, A. Barbara, J. Le Perchec, T. Lopez-Rios, Appl. Phys. Lett. 97, 053507 (2005)

12. F.J. Garcia-Vidal, J. Sanchez-Dehesa, A. Dechelette, E. Bustarret, T. Lopez-Rios, T. Fournier, B. Pannetier, J. Lightwave Technol. 17, 2191 (1999) 
13. Y. Kurokawa, H. Miyazaki, Phys. Rev. B 75, 035411 (2007)

14. C. Billaudeau, S. Collin, C. Sauvan, N. Bardou, F. Pardo, J. Pelouard, Opt. Lett. 33(2), 165 (2008)

15. C. Bonnand, J. Bellesa, C. Symonds, J. Plenet, Appl. Phys. Lett. 89, 231119 (2006)

16. T. López-Rios, D. Mendoza, F.J. García-Vidal, J. SánchezDehesa, B. Pannetier, Phys. Rev. Lett. 81, 665 (1998)

17. D. Crouse, IEEE Trans. Electr. Dev. 52, 2365 (2005)

18. D. Skigin, A. Fantino, S. Grosz, J. Opt. A: Pure Appl. Opt. 5, S129 (2003)

19. A. Barbara, P. Quémerais, E. Bustarret, T. Lopez-Rios, T. Fournier, Eur. Phys. J. D 23, 143 (2003)

20. H. Lochbihler, R. Depine, Appl. Opt. 32(19), 3459 (1993)

21. J. Le Perchec, P. Quémerais, A. Barbara, T. Lopez-Rios, Phys. Rev. Lett. 100, 066408 (2008)

22. F.J. García-Vidal, L. Martín-Moreno, Phys. Rev. B 66(15), $155412(2002)$

23. S. Collin, F. Pardo, R. Teissier, J.L. Pelouard, Phys. Rev. B 63(3), 033107 (2001)

24. P. Sheng, R.S. Stepleman, P.N. Sanda, Phys. Rev. B 26(6), 2907 (1982)

25. M.G. Moharam, D.A. Pommet, E.B. Grann, T.K. Gaylord, J. Opt. Soc. Am. A 12(5), 1077 (1995)

26. H. Ichikawa, J. Opt. Soc. Am. A 15(1), 152 (1998)

27. E. Popov, M. Nevière, S. Enoch, R. Reinisch, Phys. Rev. B. 62, $16100(2000)$

28. C. Genet, M.P.v. Exter, J.P. Woerdman, Opt. Comm. 225(4-6), 331 (2003), ISSN 0030-4018

29. E. Palik, Handbook of Optical Constants of Solids (Academic Press, San Diego, 1985)

30. P. Andrew, W. Barnes, Science 306, 1002 (2004) 